\title{
Evolution of orbital angular momentum entangled bi-photon, propagating through a turbulent atmosphere
}

\author{
Filippus S. Roux \\ CSIR National Laser Centre, PO Box 395, Pretoria 0001, South Africa
}

\begin{abstract}
Orbital angular momentum (OAM) entangled bi-photons are a resource for the higher dimensional implementation of quantum cryptography, which allows secure communication over various channels. In the case where free-space is used as communication channel the initial OAM entangled bi-photon loses some or even all of its entanglement because of the scintillation that it experiences while propagating through the turbulence in the atmosphere. This decoherence of OAM entanglement has so far only been studied for the case of weak turbulence. Unfortunately, it is the more challenging strong turbulence scenario that is relevant for the practical implementation of free-space quantum communication through the atmosphere. Using an approach that differs from previous approaches, we derive a master equation for the evolution of an OAM entangled bi-photon during propagation through turbulence. However, in our approach the equation contains a derivative with respect to the propagation distance instead of time. The principle is to consider the propagation over an infinitesimal distance of OAM basis states through a random medium. This approach allows one to include, not only the effect of turbulence of arbitrary strength, but also the effect of the inner and outer scale of the turbulence, as represented by the Tartarskii and von Karman spectra. The resulting expression can predict the rates of decoherence for arbitrary initial OAM entangled states and can be used to calculate the concurrence, which measures the amount of entanglement, as a function of propagation distance for different initial entangled OAM states.
\end{abstract}

Keywords: Infinitesimal propagation equation, entangle photons, atmospheric turbulence, orbital angular momentum, decoherence

\section{INTRODUCTION}

Lasers and micro-electronics are some of the technologies that were made possible by the advent of quantum mechanics. A new wave of quantum technologies are currently being developed, including quantum computing and quantum communication. These technologies are largely based on quantum entanglement, which is a quintessencial part of quantum mechanics that distinguishes it from classical systems.

A common challenge in these technologies is the fact that entanglement is fragile and undergoes decoherence due to interactions with its environment. Much effort has therefore gone into studying decoherence and trying to come up with ways to avoid or at least reduce its effects. In free-space quantum communication decoherence can be caused by the scattering that light experiences when propagating through a turbulent atmosphere.

Orbital angular momentum (OAM) has recently become a popular basis for entangled photon states because it allows higher dimensional representations of information in quantum systems. ${ }^{1-3}$ This is also the case with free-space quantum communication. Unfortunately, the random phase modulations, induced by the spatial fluctuation in the refractive index due to turbulence in the atmosphere, scatters the initial OAM states into other OAM states, which causes the OAM entanglement to decay.

The aim of the work reported here is to investigate this process of decoherence in OAM entanglement in a turbulent atmosphere. The effect of turbulence on classical light - scintillation - has been studied for many years. ${ }^{4}$ This has also been extended to the effect of scintillation on optical beams with specific OAM modes. ${ }^{5-9}$ On the other hand, very little work has been done on the decoherence in OAM entanglement in a turbulent atmosphere. The few studies that do exist ${ }^{10-12}$ used a simplified model of the turbulence, in that they consider 
only a single phase-only transmission function that quantifies the turbulence by a single parameter, the Fried parameter $r_{0}$. Such a model tacitly assumes that the turbulence is weak.

The approach that is followed here, as presented in Section 2, is to consider how the density operator (in terms of an OAM basis) transforms after an infinitesimal propagation through a turbulent atmosphere, which is then expressed as a differential equation with respect to propagation distance. The analysis is restricted to monochromatic optical fields under the paraxial approximation. The resulting master equation contains integrals of products of OAM momentum-space wave functions. In Section 3 these integrals are considered with the aid of the generating function for OAM modes. A simple case of qubit bi-photon states is considered in Section 4 to show how such a state decoheres during propagation. The work is summarized in Section 5.

\section{MASTER EQUATION}

After propagating over an infinitesimal distance $d z$ through a turbulent medium, the momentum-space wave function of a quantum state is transformed as follows

$$
G(\mathbf{K}, z) \stackrel{d z}{\longrightarrow} G(\mathbf{K}, z)+i \frac{d z}{2 k}\left[|\mathbf{K}|^{2} G(\mathbf{K}, z)-2 k^{2} N(\mathbf{K}, z) \star G(\mathbf{K}, z)\right]
$$

where $G(\mathbf{K}, z) \equiv\langle\mathbf{K} \mid \psi\rangle$ is the momentum-space wave function of a quantum state $|\psi\rangle$, with $\mathbf{K}$ being the twodimensional spatial frequency vector on the transverse momentum-space, $k$ is the wave number, and $\star$ represents convolution. The two-dimensional Fourier transform of the refractive index fluctuation is

$$
N(\mathbf{K}, z)=\int_{-\infty}^{\infty} \tilde{\chi}(\mathbf{k})\left[\frac{\Phi_{0}(\mathbf{k})}{\Delta_{k}^{3}}\right]^{1 / 2} \exp \left(-i k_{z} z\right) \frac{\mathrm{d} k_{z}}{2 \pi},
$$

where $\mathbf{k}$ is the full three-dimensional spatial frequency vector, $\tilde{\chi}(\mathbf{k})$ is a three-dimensional random complex spectral function, $\Delta_{k}$ is its coherence width in the frequency domain, and $\Phi_{0}(\mathbf{k})$ is the three-dimensional power spectral density of the refractive index fluctuation. The index fluctuation is a real-valued function, which implies that $\tilde{\chi}^{*}(\mathbf{k})=\tilde{\chi}(-\mathbf{k})$.

Using a OAM basis, one can express a single photon quantum state as $\rho=\sum_{m n}|m\rangle \rho_{m n}\langle n|$, where $|m\rangle \equiv$ $\left|l_{m}, p_{m}\right\rangle$ represents the OAM basis elements and $\rho_{m n}$ are the matrix elements. However, since we need to do the analysis in terms of the momentum basis each of the OAM basis elements needs to be expressed in terms of the momentum basis. As a result the density operator for a single photon has the form

$$
\rho=\sum_{m n} \int_{-\infty}^{\infty}\left|\mathbf{K}_{1}\right\rangle G_{m}\left(\mathbf{K}_{1}\right) \rho_{m n} G_{n}^{*}\left(\mathbf{K}_{2}\right)\left\langle\mathbf{K}_{2}\right| \frac{\mathrm{d}^{2} K_{1}}{4 \pi^{2}} \frac{\mathrm{d}^{2} K_{2}}{4 \pi^{2}},
$$

where $G_{m}(\mathbf{K})$ is the momentum-space wave function for OAM basis element $|m\rangle$, and $|\mathbf{K}\rangle$ denotes the momentum basis elements. The expression can be generalized to arbitrary numbers of photons to incorporate entanglement.

One can now determine how $\rho$ is transformed during an infinitesimal propagation through turbulence, by applying Eq. (1) to the density operator in Eq. (3). The resulting expression can be used to produce an expansions through repeated back substitution. In the end one performs an ensemble averaging over different random functions. The ensemble average of $\tilde{\chi}$ is zero, however the ensemble average of products of $\tilde{\chi}$ are given by

$$
\left\langle\tilde{\chi}\left(\mathbf{k}_{1}\right) \tilde{\chi}^{*}\left(\mathbf{k}_{2}\right)\right\rangle=\left(2 \pi \Delta_{k}\right)^{3} \delta_{3}\left(\mathbf{k}_{1}-\mathbf{k}_{2}\right)
$$

where $\delta_{3}(\mathbf{k})$ denotes a Dirac-delta function in three dimensions. We'll trunctate the expansion at this level.

The result can now be expressed as a master equation in the limit of small $d z$. For an entangled bi-photon quantum state, having one of the two photons propagating through turbulence, while the other propagates through free-space without turbulence, the master equation is given by

$$
\partial_{z} \rho_{m n p q}=V_{m n r s} \rho_{r s p q}+V_{p q r s} \rho_{m n r s}+L_{m n r s} \rho_{r s p q}-L_{T} \rho_{m n p q}
$$


where we sum over repeated indices $r$ and $s$. Furthermore,

$$
V_{m n p q}=\frac{i}{2 k} \int_{-\infty}^{\infty}\left(\left|\mathbf{K}_{1}\right|^{2}-\left|\mathbf{K}_{2}\right|^{2}\right) G_{p}\left(\mathbf{K}_{1}\right) G_{m}^{*}\left(\mathbf{K}_{1}\right) G_{n}\left(\mathbf{K}_{2}\right) G_{q}^{*}\left(\mathbf{K}_{2}\right) \frac{\mathrm{d}^{2} K_{1}}{4 \pi^{2}} \frac{\mathrm{d}^{2} K_{2}}{4 \pi^{2}}
$$

represents the free-space propagation of OAM modes without turbulence, while the dissipative terms caused by the turbulence are given by

$$
L_{T}=k^{2} \int_{-\infty}^{\infty} \Phi_{1}(\mathbf{K}) \frac{\mathrm{d}^{2} K}{4 \pi^{2}}
$$

with $\Phi_{1}(\mathbf{K})=\Phi_{0}\left(\mathbf{K}, k_{z}=0\right)$, and

$$
L_{m n p q}=k^{2} \int_{-\infty}^{\infty} \Phi_{1}(\mathbf{K}) W_{m p}^{*}(\mathbf{K}) W_{n q}(\mathbf{K}) \frac{\mathrm{d}^{2} K}{4 \pi^{2}}
$$

with

$$
W_{m n}(\mathbf{K})=\int_{-\infty}^{\infty} G_{m}\left(\mathbf{K}_{1}\right) G_{n}^{*}\left(\mathbf{K}_{1}-\mathbf{K}\right) \frac{\mathrm{d}^{2} K_{1}}{4 \pi^{2}}
$$

The Markov approximation is used in the derivation of the master equation given in Eq. (5). This approximation follows from the fact that the fluctuations in the refractive index is extremely small compared to the average refractive index. The distance over which the phase modulation induced by the medium becomes significant is therefore much longer than the distance over which these fluctuations have some correlation. One can therefore make the step size larger than the correlation length and ignore any 'memory' in the medium.

\section{OAM MOMENTUM-SPACE WAVE FUNCTIONS}

It is convenient to perform the calculations that involve the OAM momentum-space wave functions in terms of the generating function for the OAM basis functions. In this way the generation of particular OAM modes can be postponed till after all the calculations have been done on the generating function. The first step is to compute the two-dimensional Fourier transform to obtain the momentum space generating function. The result is given by,

$$
\Theta(\mathbf{K}, z, u, v, w)=\frac{\pi \omega_{0} N}{1+w} \exp \left\{\frac{i\left[k \omega_{0} u+i\left(k_{x}-i k_{y}\right) R(z, w)\right]\left[k \omega_{0} v+i\left(k_{x}+i k_{y}\right) R(z, w)\right]}{2 k R(z, w)(1+w)}\right\},
$$

where $\mathbf{K}=k_{x} \hat{x}+k_{y} \hat{y}, \omega_{0}$ is the initial radius of the mode profile, $N$ is a mode dependent normalization constant given by,

$$
N=\left[\frac{2^{|l|+1} p !}{\pi(|l|+p) !}\right]^{1 / 2}
$$

and $R(z, w)=(1-w) z_{R}-i(1+w) z$, with $z_{R}$ being the Rayleigh range given by $z_{R}=\pi \omega_{0}^{2} / \lambda$. This momentumspace generating function can be used to obtain an expression for the angular spectrum of any particular LaguerreGaussian mode with particular values for $p$ and $l$. This is done by taking the $l$-th derivative with respect to $u$ $(v)$ for positive (negative) $l$, taking the $p$-th derivative with respect to $w$, dividing by $p$ ! and setting $u, v$ and $w$ equal to zero.

The momentum-space generating function in Eq. (10) is now used to evaluate the integrals for Eqs. (6)(9). First we consider the integral in the free-space propagation term given in Eq. (6). One finds that it gives orthogonality conditions with respect to the azimuthal indices of the OAM modes, but not with respect to the radial indices. The latter is a result of the fact that the OAM modes depend on the propagation distance. Using the generating function one obtains the following expression

$$
V_{m n p q}=\left.\delta\left(l_{m}, l_{p}\right) \delta\left(l_{n}, l_{q}\right) \partial_{h}\left[P\left(l_{m}, p_{m}, p_{p}\right) P^{*}\left(l_{n}, p_{n}, p_{q}\right)\right]\right|_{h=0}
$$


where $\delta\left(l_{m}, l_{p}\right)$ is a Kronecker delta function, $h$ is a parameter used to generate the $\left(\left|\mathbf{K}_{1}\right|^{2}-\left|\mathbf{K}_{2}\right|^{2}\right)$-factor, and

$$
P\left(l, p_{1}, p_{2}\right)=|l| ! \sqrt{\frac{p_{1} ! p_{2} !}{\left(|l|+p_{1}\right) !\left(|l|+p_{2}\right) !}}\left[\frac{2 z_{R}}{\left(1+w_{2}\right) R\left(z, w_{1}\right)+\left(1+w_{1}\right) R^{*}\left(z, w_{2}\right)-i\left(1+w_{1}\right)\left(1+w_{2}\right) h}\right]^{1+|l|},
$$

where $w_{1}$ and $w_{2}$ are the parameters associated with the radial indices $p_{1}$ and $p_{2}$, respectively.

Next we consider the integrals for the dissipative terms given in Eqs. (7)-(9). The formalism allows one to include any spectral model $\Phi_{0}(\mathbf{k})$ for the turbulence, however, for the sake of tractibility we'll neglect the effect of the inner and outer scales. We use the von Karman spectrum, ${ }^{4}$

$$
\Phi_{0}(\mathbf{k})=\frac{0.033 C_{n}^{2}}{\left(|\mathbf{k}|^{2}+\kappa_{0}^{2}\right)^{11 / 6}}
$$

where $C_{n}^{2}$ is the refractive index structure constant for the turbulence and $\kappa_{0}$ is inversely proportional to the outer scale of the turbulence. The outer scale helps to regularize the integrals, but in the limit of large outer scale it disappears from the final expressions. Substituting Eq. (14) into Eq. (7), one finds,

$$
L_{T}=0.1244 C_{n}^{2} \kappa_{0}^{-5 / 3}+\mathrm{O}(1) .
$$

Next we evalute the correlation integrals in Eq. (9), expressing the result in polar momentum-space coordinates, so that $k_{x}+i k_{y}=K \exp (i \phi)$. For simplicity we evaluate the azimuthal indices explicitely while leaving the radial indices implicite in terms of the parameter $w_{m}$ and $w_{n}$. The asimuthal indices are then given directly by $m\left(=l_{m}\right)$ and $n\left(=l_{n}\right)$, not to be confused with the subscripts of $w_{m}$ and $w_{n}$. For $m n>0$ the resulting expression is given by

$$
W_{m, n}\left(K, \phi, z, w_{m}, w_{n}\right)=\exp \left(-\frac{X^{2} E_{m} E_{n}}{2 Q_{m n}}\right) \frac{E_{m}^{|n|} E_{n}^{|m|} e^{i(m-n) \phi}}{\sqrt{|m| !|n| !} Q_{m n}^{1+|m-n|}}\left(\frac{i X}{\sqrt{2}}\right)^{|m-n|}\left(\frac{z_{R}+i z}{z_{R}-i z}\right)^{(|m|-|n|) / 2}
$$

and for $m n \leq 0$ it is given by

$$
\begin{aligned}
W_{m, n}\left(K, \phi, z, w_{m}, w_{n}\right)= & \exp \left(-\frac{X^{2} E_{m} E_{n}}{2 Q_{m n}}\right) \frac{E_{m}^{|n|} E_{n}^{|m|} e^{i(m-n) \phi} \sqrt{|m| !|n| !}}{Q_{m n}^{1+|m+n|}}\left(\frac{i X}{\sqrt{2}}\right)^{|m+n|}\left(\frac{z_{R}+i z}{z_{R}-i z}\right)^{(|m|-|n|) / 2} \\
& \times \sum_{r=0}^{M} \frac{\left(-\frac{2 Q_{m n}}{X^{2} E_{m} E_{n}}\right)^{r}}{r ! \Gamma(|m|-r+1) \Gamma(|n|-r+1)}
\end{aligned}
$$

where

$$
X=\frac{K \sqrt{z^{2}+z_{R}^{2}}}{k \omega_{0}} \quad E_{m}=1-w_{m}\left(\frac{z_{R}+i z}{z_{R}-i z}\right) \quad E_{n}=1-w_{n}\left(\frac{z_{R}-i z}{z_{R}+i z}\right) \quad Q_{m n}=1+w_{m} w_{n} .
$$

It is challenging to express the result for the integral in Eq. (8) as a single expression. However, one can determine what the respective expressions for the different element would be by considering the integral term-byterm. This is further aleviated by the following two observations. In the first place one notices that $\Phi_{1}(\mathbf{K})$ only depends on the radial coordinate and not the angle. Therefore the integral over $\phi$ only include the $\phi$-dependences of Eqs. (16) and (17), which is given by $\exp [i(n-q-m+p) \phi]$. The result of this angular integral is therefore zero unless $n-q-m+p=0$. As a result many of the elements in $L_{m n p q}$ vanish.

The second observation is that, as far as the $K$-dependence is concerned, those elements that survive the $\phi$-integration always consist of terms of the form

$$
f_{m}(K)=\frac{A \exp \left(-B K^{2}\right) K^{2 m}}{\left(K^{2}+\kappa_{0}^{2}\right)^{11 / 6}}
$$


where $m$ is a non-negative integer, $A$ contains all the multiplicative parameters from Eqs. (14), (8), (16) and (17), and $B$ is a parameter composed of the parameters in the exponent of Eq. (16) or (17). If $m=0$ the integral over $f_{m}(K)$ diverges as $\kappa_{0} \rightarrow 0$. In the limit of small $\kappa_{0}$ the leading terms are,

$$
\int_{-\infty}^{\infty} f_{0}(K) K \mathrm{~d} K \approx L_{T}-\frac{C_{n}^{2} \pi^{1 / 2} \omega_{0}^{5 / 3}\left(1+t^{2}\right)^{5 / 3}}{6 \Gamma(2 / 3)}
$$

where $t=z / z_{R}$ and $L_{T}$ is given by Eq. (15). It turns out that the $L_{T}$-term in Eq. (5) exactly cancel off all the $L_{T}$-terms that appear inside the $L_{m n p q}$-term in Eq. (5) as a result of Eq. (20).

The integrals of $f_{m}(K)$ with $m>0$ all give finite results independent of $\kappa_{0}$ in the limit where $\kappa_{0} \rightarrow 0$. For instance, if the radial index is zero $(p=0)$ the leading term has the form,

$$
\int_{-\infty}^{\infty} f_{m}(K) K \mathrm{~d} K \approx \frac{G_{m} C_{n}^{2} \pi^{1 / 2} \omega_{0}^{5 / 3}\left(1+t^{2}\right)^{5 / 3}}{\Gamma(2 / 3)}
$$

where $G_{m}$ is a numerical constant that only depends on $m$. One can combine all the appropriate terms to obtain expressions for the elements of $L_{m n p q}$ and then use them to obtain the explicit expression for the master equation in Eq. (5).

Since it represents an infinite set of coupled first-order differential equations, the master equation in Eq. (5) is not easy to solve in general. Assuming that the intermodal coupling decreases for higher order modes, one can truncate the set of equations. Next we consider such a trunctated case.

\section{EXAMPLE: QUBIT BI-PHOTON}

As an example, we consider the case ${ }^{10}$ where only modes with the lowest radial index $(p=0)$ and with azimuthal indices of the same magnitude $l= \pm q(q=1,2,3)$ are retained. This truncation implies that much of the coupling with higher order modes is lost. The trace of the truncated density matrix is not equal to 1 anymore. We shall use the value of the trace of the truncated density matrix to provide an indication of the loss of information to the higher order modes.

For this case $V_{m n p q}(z)=0$ and the only nonzero elements of $L_{m n r s}$ are

$$
\begin{aligned}
L_{q, q, q, q}(z)=L_{q,-q, q,-q}(z)=L_{-q, q,-q, q}(z)=L_{-q,-q,-q,-q}(z) & =L_{T}-A_{q} h(z) \\
L_{q, q,-q,-q}(z)=L_{-q,-q, q, q}(z) & =B_{q} h(z),
\end{aligned}
$$

where $A_{q}$ and $B_{q}$ are positive constants that only depend on $q,{ }^{*}$ and $h(z)$ is the same function for all the terms. It contains all the dimension parameters

$$
h(z)=\frac{1}{z_{R}}\left(C_{n}^{2} \omega_{0}^{2 / 3}\right)\left(\frac{\lambda}{\pi \omega_{0}}\right)^{-3}\left(1+\frac{z^{2}}{z_{R}^{2}}\right)^{5 / 6} .
$$

Provided that the turbulence is not too weak and that the beam waist is not too small, the entanglement decays to zero over a distance much shorter than the Rayleigh range, which allows one to assume that $1+z^{2} / z_{R}^{2} \approx 1$. Under these circumstances one can express the integral of $h(z)$ in terms of the Fried parameter $r_{0}$,

$$
\int_{0}^{z} h\left(z^{\prime}\right) \mathrm{d} z^{\prime}=0.592\left(\frac{\omega_{0}}{r_{0}}\right)^{5 / 3}
$$

where $r_{0}=0.185\left(\lambda^{2} / C_{n}^{2} / z\right)^{3 / 5}$. As a result all the dimension parameters are combined into $\omega_{0} / r_{0}$.

\footnotetext{
${ }^{*}$ The explicite values are: $A_{1}=1.570, A_{2}=2.206, A_{3}=2.807$ and $B_{1}=0.03030, B_{1}=0.004787, B_{1}=0.001754$.
} 


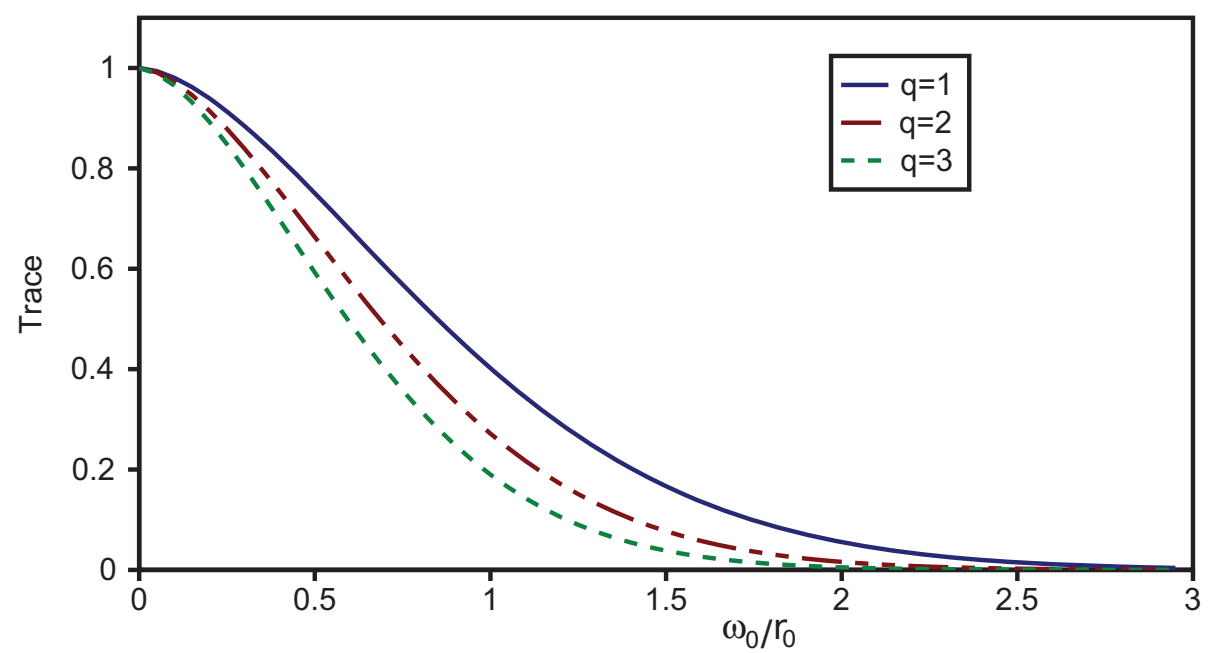

Figure 1. Plots of the trace of the density matrix for the entangled bi-photon, initially in the singlet Bell-state, in terms of two OAM states with $l= \pm q$, for $q=1,2,3$, as a function of $\omega_{0} / r_{0}$.

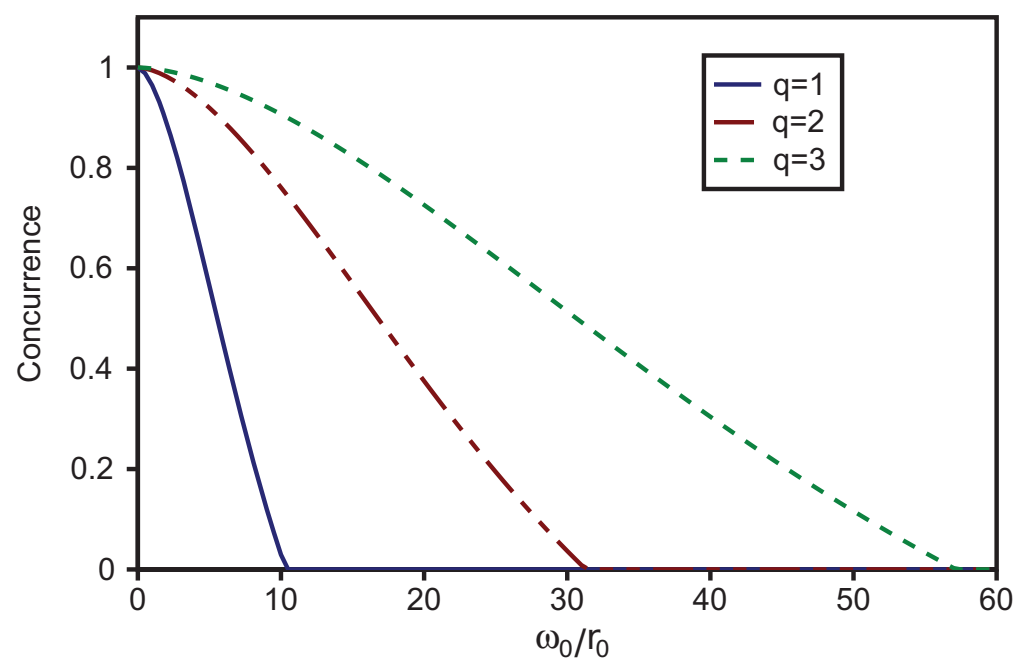

Figure 2. Plots of the concurrence for a bi-photon, initially in the singlet Bell-state, in terms of two OAM states with $l= \pm q$, for $q=1,2,3$, as a function of $\omega_{0} / r_{0}$.

Assuming that the initial state of the density matrix is the singlet Bell-state in the OAM basis, one obtains the following solution of the density matrix

$$
\rho_{m n p q}=\frac{T}{4}\left[\begin{array}{cccc}
1-R^{2} & 0 & 0 & 0 \\
0 & 1+R^{2} & -2 R & 0 \\
0 & -2 R & 1+R^{2} & 0 \\
0 & 0 & 0 & 1-R^{2}
\end{array}\right]
$$

where $m p(n q)$ denote the row (column) indices, and where

$$
\begin{aligned}
T & =\exp \left[-\left(A_{q}-B_{q}\right) \int_{0}^{z} h\left(z^{\prime}\right) \mathrm{d} z^{\prime}\right] \\
R & =\exp \left[-B_{q} \int_{0}^{z} h\left(z^{\prime}\right) \mathrm{d} z^{\prime}\right] .
\end{aligned}
$$


The eigenvalues of the density matrix are

$$
\lambda_{\rho}=\left\{\frac{1}{4} T(1+R)^{2}, \frac{1}{4} T(1-R)^{2}, \frac{1}{4} T\left(1-R^{2}\right), \frac{1}{4} T\left(1-R^{2}\right)\right\}
$$

which are all positive. The trace of the density matrix is given by $T$, which is a decaying function, since $A_{q}>B_{q}$. We plot the trace $T$ as a function of $\omega_{0} / r_{0}$ in Fig. 1 for $q=1,2,3$.

Using the normalized density matrix [by setting $T=1$ in Eq. (26)], we compute the concurrence of formation $^{13,14}$ and obtain

$$
\mathcal{C}=\frac{1}{2}\left(2 R+R^{2}-1\right)
$$

which is plotted in Fig. 2 for $q=1,2,3$ as a function of $\omega_{0} / r_{0}$.

From the curves for the trace in Fig. 1 one can see that modes with higher OAM are scattered more rapidly into other modes than those with lower OAM. On the other hand, from Fig. 2 we see that modes with higher OAM retain their entanglement for longer distances than those with lower OAM. These conclusions agree qualitatively with previous work, ${ }^{10}$ however, while the scattering into other modes occurs at a scale where $r_{0} \approx \omega_{0}$, similar to what was found before,${ }^{10}$ the entanglement lasts for at least an order of magnitude longer, which is quantitatively different from what was found before. ${ }^{10}$ Here the slowness of the decay in the concurrence is a result of the smallness of the values of the $B_{q}$ 's. From these results it appears that the effect of scattering and the implied loss of photons in the desired OAM modes may turn out to be a more significant challenge for free-space quantum communication than the decoherence of OAM entanglement.

\section{SUMMARY}

We derived a master equation for the decoherence experienced by an OAM entangled photon state propagating through a turbulent atmosphere. It is assumed that the light is monochromatic and propagates as a paraxial beam, and that the refractive index fluctuations are small enough to allow a Markov approximation. The formalism does not require any simplification to the turbulence model, however to compare the results with previous analyses of this case the inner and outer scales of the turbulence are ignored in the example that is considered. The result was found to be qualitatively similar to previous work, but significant quantitative differences exist.

The master equation represent an infinite set of first order differential equations. To solve such a set one would in general need to truncate the set, which could loose significant contributions of the coupling to higher order modes.

\section{ACKNOWLEDGMENTS}

The author gives thanks to Hermann Uys, Thomas Konrad, Andrew Forbes and Francesco Petruccione for fruitful discussions on this topic. This work was done with the support of an SRP Type A grant from the CSIR.

\section{REFERENCES}

[1] Mair, A., Vaziri, A., Weihs, G., and Zeilinger, A., "Entanglement of the orbital angular momentum states of photons," NATURE 412, 313-316 (2001).

[2] Bourennane, M., Karlsson, A., and Björk, G., "Quantum key distribution using multilevel encoding," Phys. Rev. A 64, 012306 (2001).

[3] Molina-Terriza, G., Torres, J. P., and Torner, L., "Management of the angular momentum of light: Preparation of photons in multidimensional vector states of angular momentum," Phys. Rev. Lett. 88, 013601 (2001).

[4] Andrews, L. C. and Phillips, R. L., [Laser Beam Propagation Through Random Media], SPIE, Washington (1998). 
[5] Gibson, G., Courtial, J., Padgett, M., Vasnetsov, M., Pas'ko, V., Barnett, S., and Franke-Arnold, S., "Freespace information transfer using light beams carrying orbital angular momentum," Opt. Express 12(22), 5448-5456 (2004).

[6] Paterson, C., "Atmospheric turbulence and orbital angular momentum of single photons for optical communication," Phys. Rev. Lett. 94, 153901 (Apr 2005).

[7] Gbur, G. and Tyson, R. K., "Vortex beam propagation through atmospheric turbulence and topological charge conservation," J. Opt. Soc. Am. A 25, 225-230 (2008).

[8] Cheng, W., Haus, J. W., and Zhan, Q., "Propagation of vector vortex beams through a turbulent atmosphere," Opt. Express 17, 17829-17836 (2009).

[9] Dipankar, A., Marchiano, R., and Sagaut, P., "Trajectory of an optical vortex in atmospheric turbulence," Phys. Rev. E 80, 046609 (Oct 2009).

[10] Smith, B. J. and Raymer, M. G., "Two-photon wave mechanics," Phys. Rev. A 74, 062104 (Dec 2006 ).

[11] Jha, A. K., Tyler, G. A., and Boyd, R. W., "Effects of atmospheric turbulence on the entanglement of spatial two-qubit states," Phys. Rev. A 81, 053832 (May 2010).

[12] Gopaul, C. and Andrews, R., "The effect of atmospheric turbulence on entangled orbital angular momentum states," New Journal of Physics 9, 94 (2007).

[13] Hill, S. and Wootters, W. K., "Entanglement of a pair of quantum bits," Phys. Rev. Lett. 78, 5022-5025 (Jun 1997).

[14] Wootters, W. K., "Entanglement of formation of an arbitrary state of two qubits," Phys. Rev. Lett. 80, 2245-2248 (Mar 1998). 